\title{
PERFIL DOS ESTUDOS DE AVALIAÇÃO DA ASSISTENCIA NA ATENÇÃO PRIMÁRIA AO USUARIO PORTADOR DE HIPERTENSÃO ARTERIAL
}

\section{PROFILE OF ASSESSMENT ASSESSMENT STUDIES IN PRIMARY CARE TO USERS WITH ARTERIAL HYPERTENSION}

Rayane Saraiva Felix ${ }^{1}$ Rayla Patrícia da Silva Andrade Soares ${ }^{2}$ Camila Priscila Abdias do Nascimento ${ }^{3}$ Alany Carla de Sousa Paiva ${ }^{4}$ Sandy Yasmine Bezerra e Silva ${ }^{5}$ Érika Simone Galvão Pinto ${ }^{6}$

RESUMO: OBJETIVO: descrever o perfil dos estudos de avaliação da assistência na atenção primária ao usuário portador de hipertensão arterial produzidos no Brasil, enfatizando principalmente o desenho metodológico adotado e características-chave de avaliação. MÉTODO: trata-se de um estudo bibliográfico, descritivo e quantitativo. A busca pelos estudos foi realizada no mês de janeiro de 2020, através da Biblioteca Digital Brasileira de Teses e Dissertações. Os critérios de inclusão utilizados foram: estudos nacionais, publicados nos últimos dez anos (2009 a 2019), disponíveis para o acesso gratuito, e na íntegra. Foram encontrados 105 estudos e após a análise completa foram selecionados os estudos cujos objetivos eram avaliar, resultando em 10 para integrarem a amostra. RESULTADOS: foi possível observar que a maioria dos trabalhos foram defendidos no período entre 2017 e 2019 (50\%). Em relação ao tipo de estudo $60 \%$ deles foram dissertações. Quanto a metodologia aplicada $70 \%$ dos estudos tem abordagem quantitativa, do tipo descritivo (50\%).

\footnotetext{
1 Enfermeira. Mestranda pelo Programa de Pós-Graduação em Enfermagem (PPGENF) da Universidade Federal do Rio Grande do Norte- UFRN, rsf1601@hotmail.com;

2 Enfermeira. Mestranda pelo Programa de Pós-Graduação em Enfermagem (PPGENF) da Universidade Federal do Rio Grande do Norte- UFRN, raylapatriciayla@gmail.com;

3 Enfermeira. Mestranda pelo Programa de Pós-Graduação em Enfermagem (PPGENF) da Universidade Federal do Rio Grande do Norte- UFRN, camilapriabd@gmail.com;

4 Enfermeira. Mestranda pelo Programa de Pós-Graduação em Enfermagem (PPGENF) da Universidade Federal do Rio Grande do Norte- UFRN, alanycarla@hotmail.com;

${ }^{5}$ Enfermeira. Doutoranda em Enfermagem pelo Programa de Pós-Graduação em Enfermagem (PPGENF) da Universidade Federal do Rio Grande do Norte - UFRN, sandyyasmine@hotmail.com;

${ }^{6}$ Enfermeira. Prof. ${ }^{a}$ Dra. do Programa de Pós-Graduação do Mestrado Acadêmico em Enfermagem da Universidade Federal do Rio Grande do Norte - UFRN, erikasgp@gmail.com.
} 
Quanto ao tipo de avaliação obteve-se a mesma quantidade para a Avaliação Normativa (50\%) e Pesquisa Avaliativa (50\%). Quanto aos estudos de Pesquisa Avaliativa, em $60 \%$ deles foi realizada uma análise de efeito. CONCLUSÃO: a realização do presente artigo proporcionou um aprofundamento da aprendizagem sobre os modelos de avaliação e percurso metodológico utilizados nos estudos de avaliação em saúde nos últimos anos, tendo sido de importância significativa para o aprimoramento dos conhecimentos com relação à avaliação dos serviços de saúde.

Palavras chave: Atenção Primária à saúde. Avaliação em Saúde. Hipertensão.

ABSTRACT: OBJECTIVE: to describe the profile of studies evaluating care in primary care for patients with arterial hypertension produced in Brazil, emphasizing mainly the methodological design adopted and key evaluation characteristics. METHOD: This is a bibliographic, descriptive and quantitative study. The search for studies was carried out in January 2020, through the Brazilian Digital Library of Theses and Dissertations. The inclusion criteria used were: national studies, published in the last ten years (2009 to 2019), available for free access, and in full. 105 studies were found and after the complete analysis, studies whose objectives were to be evaluated were selected, resulting in 10 to integrate the sample. RESULTS: It was possible to observe that most works were defended in the period between 2017 and 2019 (50\%). Regarding the type of study, 60\% of them were dissertations. As for the methodology applied, $70 \%$ of the studies have a quantitative approach, of the descriptive type (50\%). As for the type of evaluation, we had the same amount for Normative Evaluation (50\%) and Evaluative Research (50\%). As for the Evaluative Research studies, in $60 \%$ of them an effect analysis was performed. CONCLUSION: The realization of this article provided a deepening of learning about the evaluation models and methodological path used in health evaluation studies in recent years, having been of significant importance for the improvement of knowledge regarding the evaluation of health services.

Keywords: Primary Health Care. Health Evaluation. Hypertension. 


\section{INTRODUÇÃO}

A Hipertensão Arterial Sistêmica (HAS) representa um sério problema epidemiológico no Brasil, tanto pela sua elevada prevalência na população adulta e idosa, quanto pelas complicações que acarreta, com acentuadas taxas de morbimortalidade e impactos relevantes nos custos hospitalares, previdenciários, econômicos e sociais. (MALTA et al, 2018).

A Organização Mundial da Saúde (OMS) estima que cerca de 600 milhões de pessoas tenham HAS, com crescimento global de $60 \%$ dos casos até 2025 , além de cerca de 7,1 milhões de mortes anuais. No Brasil, a HAS atinge 32,5\% (36 milhões) de indivíduos adultos, mais de $60 \%$ dos idosos, contribuindo direta ou indiretamente para $50 \%$ das mortes por Doença Cardiovascular (DCV). (SOCIEDADE BRASIELIRA DE CARDIOLOGIA, 2016).

No Brasil, os desafios do controle e prevenção da HAS e suas complicações são, sobretudo, das equipes que prestam Atenção Primária em Saúde (APS). Essas equipes são multiprofissionais, cujo processo de trabalho pressupõe vínculo com a comunidade e a clientela adstrita, levando em conta a diversidade racial, cultural, religiosa e os fatores sociais envolvidos. (BRASIL, 2014).

De acordo com Starfield (2004) a APS é reconhecidamente um componentechave dos sistemas de saúde. Esse reconhecimento fundamenta-se nas evidências de seu impacto na saúde e no desenvolvimento da população nos países que a adotaram como base para seus sistemas de saúde, pois tem a capacidade de desenvolver: melhores indicadores de saúde, maior eficiência no fluxo dos usuários dentro do sistema, tratamento mais efetivo de condições crônicas, maior eficiência do cuidado, maior utilização de práticas preventivas, maior satisfação dos usuários e diminuição das iniquidades sobre o acesso aos serviços e o estado geral de saúde.

Ainda de acordo com Starfield (2004) a APS apresenta dois aspectos distintos e interdependentes: é uma estratégia de organização e reorganização dos 
sistemas de saúde, nos quais representa o primeiro nível de atenção, e um modelo de mudança da prática clínico- assistencial dos profissionais de saúde. Orienta-se por eixos estruturantes que, na literatura internacional, recebem o nome de atributos essenciais: atenção ao primeiro contato, longitudionalidade, integralidade e coordenação; e atributos derivados: orientação familiar e comunitária e competência cultural.

No Brasil a organização dos serviços de saúde na APS ocorre por meio da Estratégia de Saúde da Família (ESF), que prioriza as ações de promoção, proteção e recuperação de saúde, de forma integral e continuada. Em expansão por todo o território nacional, a ESF define-se por um conjunto de ações e serviços que vão além da assistência médica, estruturando-se com base no reconhecimento das necessidades da população, apreendidas a partir do estabelecimento de vínculos entre os usuários dos serviços e os profissionais de saúde, em contato permanente com o território. (MACINKO; MENDONÇA, 2018).

O reconhecido potencial desse nível de assistência a saúde e a sua expansão acentuada a partir de 1994, colocou no centro das discussões questionamentos relacionados à eficiência, resolutividade e capacidade de articulação de seus serviços com os demais níveis de atenção. Em resposta a essas demandas, o Ministério da Saúde (MS), em parceria com outras instituições, vem fomentando um conjunto de estratégias para o monitoramento e a avaliação dos serviços de APS, com vistas a ampliar o acesso e a qualidade, além de desenvolver nesses espaços a cultura da avaliação como prática institucional de acompanhamento e gestão (BRASIL, 2015).

A avaliação da qualidade no âmbito da APS constitui tema relevante nas últimas décadas e incitando, em instituições acadêmicas e nos serviços, o desenvolvimento e a utilização de uma diversidade de princípios, técnicas e instrumentos (PINTO JÚNIOR ET AL., 2015).

A avaliação da APS é uma tarefa que exige os esforços e a participação de diversas instituições e profissionais, dada a sua magnitude, complexidade e heterogeneidade. Assim, avaliar as diferentes perspectivas dos serviços e o grau de orientação de cada um desses espaços possibilita a produção rigorosa de conhecimentos acerca de sua efetividade e diversidade (BRASIL, 2010). 
Dessa forma, este trabalho tem o objetivo de descrever o perfil dos estudos de avaliação da assistência na atenção primária ao usuário portador de hipertensão arterial produzidos no Brasil, enfatizando principalmente o desenho metodológico adotado e características-chave de avaliação.

\section{METODOLOGIA}

Trata-se de um estudo de revisão bibliográfica, descritivo e quantitativo. A busca pelos estudos realizou-se no mês de janeiro de 2020, através da Biblioteca Digital Brasileira de Teses e Dissertações. A busca pelos estudos considerou os seguintes descritores e os conectores boleanos: Avaliação em Saúde and Atenção Primária à saúde and Hipertensão. Como critério de inclusão utilizamos: estudos nacionais, publicados nos últimos dez anos (2009 a 2019), disponíveis para acesso gratuito, na íntegra.

Foram encontrados 105 estudos, três foram excluídos por serem duplicados. Após a leitura de títulos e dos resumos foram selecionados 15 estudos. Por fim foi realizada a análise completa, cuja etapa selecionaria os estudos cujos objetivos eram avaliar, resultando em 10 estudos para integrarem a amostra. (Figura 1). Por ser tratar de pesquisa com dados públicos de fonte secundária o estudo não foi submetido ao Comitê de Ética em Pesquisa. 
Figura 1. Fluxograma de busca dos estudos na Biblioteca Digital Brasileira de Teses e Dissertações.

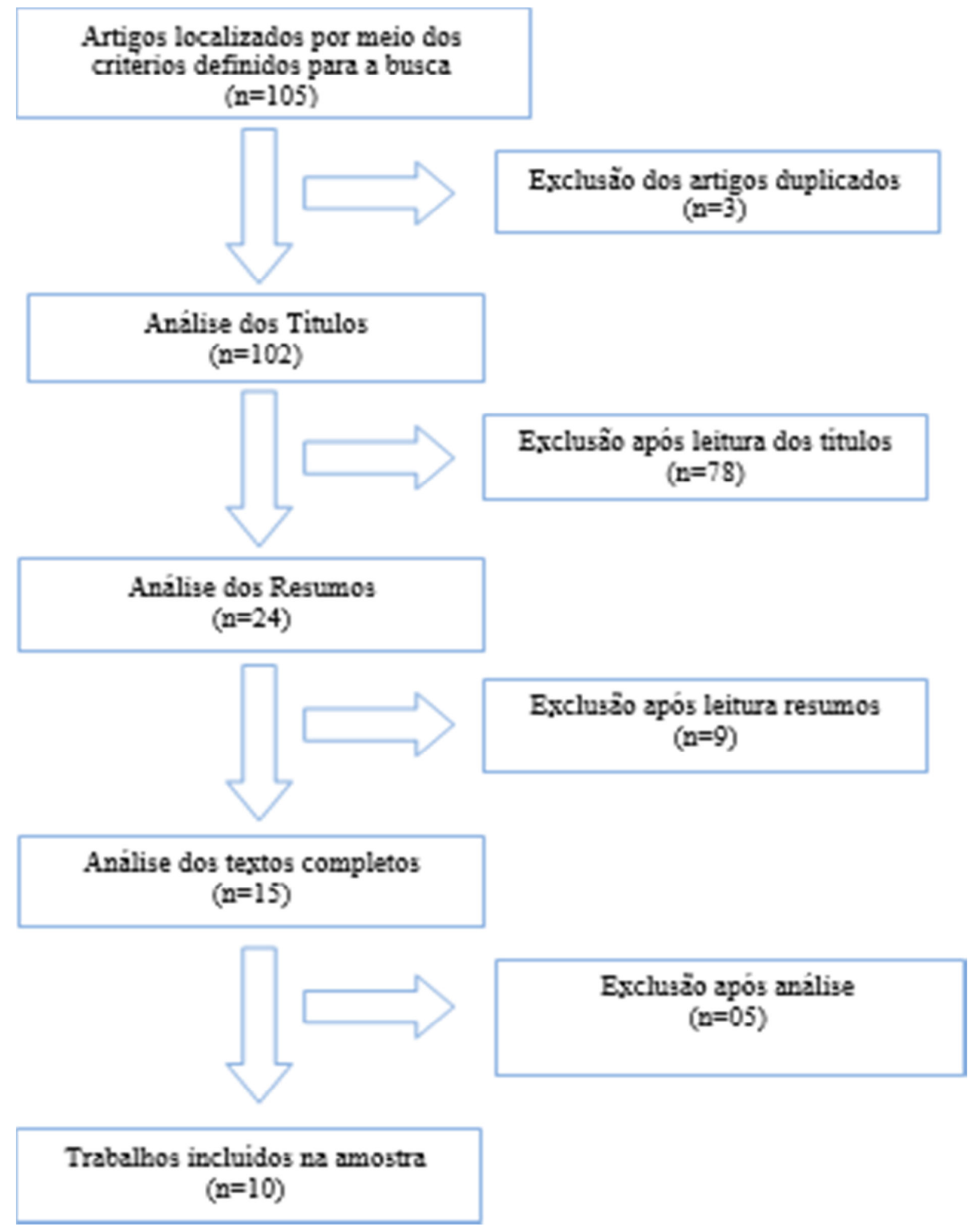

Fonte: Autores. 


\section{RESULTADOS}

Dos trabalhos incluídos nesta revisão, foram extraídas as seguintes informações: Autoria, Ano de defesa, Tipo de estudo (Quadro 1), além de e Metodologia utilizada e Tipos de avaliação (Quadro 2).

Quadro 1: Informações sobre autoria, ano de defesa e tipo dos estudos selecionados.

\begin{tabular}{|c|c|c|}
\hline Autoria & Ano de defesa & Tipo de estudo \\
\hline REGO, A. S & 2017 & Dissertação \\
\hline SILVA, L. B & 2018 & Tese \\
\hline PUGLISI, M. A & 2019 & Dissertação \\
\hline SANTOS, J. M. M & 2018 & Dissertação \\
\hline FIRMINO, P. Y. M & 2017 & Tese \\
\hline SILVA, C. S & 2015 & Tese \\
\hline RABETTI, A. C & 2009 & Dissertação \\
\hline TRINDADE, T. G & 2013 & Tese \\
\hline MACEDO, C.L. S. V & 2013 & Dissertação \\
\hline MELO, E.C. A & 2011 & Dissertação \\
\hline
\end{tabular}

Fonte: Autores.

Ao analisarmos o Quadro 1 pode-se observar que a maioria dos trabalhos foram defendidos no período entre 2017 e 2019 (50\%). Em relação ao tipo de estudo $60 \%$ deles foram dissertações. Quanto a metodologia aplicada $70 \%$ dos estudos tem abordagem quantitativa, do tipo descritivo (50\%). 
Quadro 2: Informações sobre a metodologia e o tipo de avaliação dos estudos selecionados.

\begin{tabular}{|c|c|c|}
\hline Autoria & Metodologia & Tipo de Avaliação \\
\hline REGO, A. S & $\begin{array}{l}\text { Estudo Transversal-descritivo, } \\
\text { abordagem quantitativa. }\end{array}$ & $\begin{array}{l}\text { Pesquisa Avaliativa } \\
\text { (Análise de Efeito) }\end{array}$ \\
\hline SILVA, L. B & $\begin{array}{l}\text { Estudo de métodos mistos com desenho } \\
\text { sequencial explanatório. }\end{array}$ & $\begin{array}{l}\text { Pesquisa Avaliativa } \\
\text { (Análise de Efeito) }\end{array}$ \\
\hline PUGLISI, M. A & $\begin{array}{l}\text { Estudo avaliativo de abordagem quantitativa, } \\
\text { transversal. }\end{array}$ & $\begin{array}{l}\text { Avaliação } \\
\text { Normativa }\end{array}$ \\
\hline SANTOS, J. M. M & $\begin{array}{l}\text { Estudo exploratório, com abordagem } \\
\text { quantitativa e qualitativa. }\end{array}$ & $\begin{array}{l}\text { Avaliação } \\
\text { Normativa }\end{array}$ \\
\hline FIRMINO, P & $\begin{array}{l}\text { Estudo descritivo, com abordagem } \\
\text { quantitativa e qualitativa. }\end{array}$ & $\begin{array}{l}\text { Avaliação } \\
\text { Normativa }\end{array}$ \\
\hline SILVA, C. S & $\begin{array}{l}\text { Estudo observacional, tipo coorte única, } \\
\text { retrospectivo e de base populacional com } \\
\text { abordagem quantitativa. }\end{array}$ & $\begin{array}{l}\text { Pesquisa Avaliativa } \\
\text { (Análise de } \\
\text { Intervenção) }\end{array}$ \\
\hline RABETTI, A. C & $\begin{array}{l}\text { Estudo avaliativo, transversal, } \\
\text { abordagem quantitativa }\end{array}$ & $\begin{array}{l}\text { Pesquisa Avaliativa } \\
(\text { Análise de } \\
\text { Rendimento) }\end{array}$ \\
\hline TRINDADE, T. G & $\begin{array}{l}\text { Estudo Transversal-descritivo, comF } \\
\text { abordagem quantitativa. }\end{array}$ & $\begin{array}{l}\text { Pesquisa Avaliativa } \\
\text { (Análise de Efeito) }\end{array}$ \\
\hline $\begin{array}{l}\text { MACEDO, C. L. S } \\
\text { V }\end{array}$ & $\begin{array}{l}\text { Estudo avaliativo, descritivo e transversal } \\
\text { com abordagem quantitativa. }\end{array}$ & $\begin{array}{l}\text { Avaliação } \\
\text { Normativa }\end{array}$ \\
\hline MELO, E. C. A & $\begin{array}{l}\text { Estudo transversal, de caráter descritivo, de } \\
\text { avaliação de serviços de saúde com } \\
\text { abordagem quantitativa. }\end{array}$ & \\
\hline
\end{tabular}

Fonte: Autores.

No quadro 2 é possível observar que quanto ao tipo de avaliação obteve-se a mesma quantidade para a Avaliação Normativa (50\%) e Pesquisa Avaliativa (50\%). Quanto aos estudos de Pesquisa Avaliativa, em 60\% deles foi realizada uma análise de efeito.

\section{DISCUSSÃO}

A análise dos dados permitiu verificar que os estudos de avaliação em saúde realizados por alunos de pós-graduação estão cada vez mais numerosos. Este 
resultado é positivo pois a avaliação constitui um instrumento essencial de apoio à gestão.

De acordo com Contandriopoulos, et al (1997), avaliar consiste fundamentalmente em fazer um julgamento de valor a respeito de uma intervenção ou sobre qualquer um dos seus componentes com o objetivo de ajudar na tomada de decisão. Nesse sentido, o papel da avaliação no processo de gestão é o de fornecer elementos de conhecimento que subsidiem a tomada de decisão, propiciando o aumento da eficiência, eficácia e efetividade das atividades desenvolvidas pelo serviço ou pela organização.

Para Furtado (2014) a concepção do processo avaliativo tem que estar sintonizada com o contexto do objeto a ser avaliado, com as necessidades de saúde da população, com as escolhas do problema de saúde, com a identificação dos atores que têm poder de tomar as decisões, com os que têm a capacidade técnica de implementá-las e com as políticas, estratégias e prioridades do setor saúde.

Quando observa-se a metodologia utilizada pelos estudos que compõem a amostra, tem-se em sua maioria estudos com abordagem quantitativa do tipo descritivo. Segundo Tanaka (2017) o avaliador tem de conceber um processo avaliativo através da escolha de metodologias, indicadores e de parâmetros que contemple essa diversidade de aspectos e que direcione as decisões e as ações para propiciar o máximo de benefício à saúde da população. Uma tarefa que faz com que cada avaliação seja um caso particular que requer criatividade por parte do investigador/avaliador na formulação da melhor estratégia, na seleção da abordagem, na definição de níveis e atributos, bem como na seleção de critérios, indicadores e padrões.

Neste estudo, também observou-se que quando falamos de tipo de avaliação o resultado foi diverso, com a presença de estudo de avaliação normativa e pesquisas avaliativas com análises de efeito, rendimento e intervenção.

De acordo com Contandriopoulos, et al (1997) a avaliação normativa é a atividade que consiste em fazer um julgamento sobre uma intervenção, comparando os recursos empregados e sua organização (estrutura), os serviços ou os bens produzidos (processo), e os resultados obtidos, com critérios e normas, já a 
pesquisa avaliativa é o procedimento que consiste em fazer um julgamento ex-post de uma intervenção usando métodos científicos.

Santos e Silva (2017) expõem que, apesar do entrelaçamento existente entre a avaliação de programas (avaliação normativa) e a pesquisa avaliativa, existem diferenças quanto à natureza de ambas, a avaliação normativa consiste na elaboração de normas e critérios para análise de programas, enquanto a pesquisa avaliativa utiliza procedimento científico para averiguar os componentes de um programa governamental.

\section{CONSIDERAÇÕES FINAIS}

Foram incluídos nesta revisão dez estudos após análise. Dos artigos incluídos $60 \%$ deles foram dissertações. Em relação ao tipo de avaliação obteve-se a mesma quantidade para a Avaliação Normativa (50\%) e Pesquisa Avaliativa (50\%). Quanto aos estudos de Pesquisa Avaliativa, em $60 \%$ deles foi realizada uma análise de efeito.

A realização do presente artigo proporcionou um aprofundamento da aprendizagem sobre os modelos de avaliação e percurso metodológico utilizados nos estudos de avaliação em saúde nos últimos anos, tendo sido de importância significativa para o aprimoramento dos conhecimentos com relação à avaliação dos serviços de saúde.

Neste sentido, conclui-se que a avaliação em saúde é uma importante ferramenta para acompanhamento e monitoramento das ações com objetivo de subsidiar melhorias e elaboração de políticas públicas, tendo em vista que traz uma análise da realidade e a viabilidade dos recursos e serviços a serem ofertados. 


\section{REFERÊNCIAS BIBLIOGRÁFICAS}

BRASIL. Ministério da Saúde. Secretaria de Atenção em Saúde. Departamento de Atenção Básica. Manual do instrumento de avaliação da atenção primária à saúde: primarycare assessment tool pcatool - Brasil / Ministério da Saúde, Secretaria de Atenção em Saúde, Departamento de Atenção Básica. - Brasília: Ministério da Saúde, 2010.

BRASIL. Ministério da Saúde. Secretaria de Atenção à Saúde. Departamento de Atenção Básica. Estratégias para o cuidado da pessoa com doença crônica: hipertensão arterial sistêmica / Ministério da Saúde, Secretaria de Atenção à Saúde, Departamento de Atenção Básica. - Brasília: Ministério da Saúde, 2014.

CONTANDRIOPOULOS, A. P.; CHAMPAGNE, F.; DENIS, J. L. \& PINEAULT, R., 1997. A avaliação na área de saúde: Conceitos e métodos. In: Avaliação em Saúde: Dos Modelos Conceituais à Prática na Análise da Implantação de Programas (Z. M. A. Hartz, org.), pp. 29-47, Rio de Janeiro: Editora Fiocruz, 1997.

FURTADO, J. P. A avaliação de programas e serviços de saúde no Brasil enquanto espaço de saberes e práticas. Cad. Saúde Pública, Rio de Janeiro, 30(12):2643-2655, dez, 2014.

MACINKO, J.; MENDONÇA, C. S. Estratégia Saúde da Família, um forte modelo de Atenção Primária à Saúde que traz resultados. Saúde Debate, Rio de Janeiro, v. 42, n. (spe1), p. 18-37, set 2018.

MALTA, D. C. et al. Prevalência da hipertensão arterial segundo diferentes critérios diagnósticos, Pesquisa Nacional de Saúde. Revista Brasileira de Epidemiologia, v. $21 \mathrm{n}$. (SUPL), P. 1-15, dez. 2018.

PINTO JÚNIOR, E. P. et al. Análise da produção científica sobre avaliação, no contexto da saúde da família, em periódicos brasileiros. Saúde em Debate, Rio de Janeiro, v. 39, n. 104, p. 268-278, 2015.

SANTOS, L. C. F.; SILVA, S. A. M. Valiação da Avaliação de Programas Governamentais: uma proposta de meta-avaliação dos resultados da pesquisa avaliativa da Ouvidoria-Geral do SUS. IV Encontro Brasileiro de Administração Pública, 2017.

SOCIEDADE BRASILEIRA DE CARDIOLOGIA, SOCIEDADE BRASILEIRA DE HIPERTENSÃO E SOCIEDADE BRASILEIRA DE NEFROLOGIA.V Diretrizes Brasileiras de Hipertensão Arterial. São Paulo, v. 5. 2016.

STARFIELD, B. Atenção primária: equilíbrio entre necessidade de saúde, serviços e tecnologia. Brasília: UNESCO: Ministério da Saúde, 2004.

TANAKA, O. Y. Avaliação em saúde: novos tempos, novas construções. In: TANAKA, O. Y.; RIBEIRO, E. L.; ALMEIDA, C. A. L. Avaliação em saúde: contribuições para incorporação no cotidiano. Rio de Janeiro: Atheneu, 2017. p. 1-9. 